\title{
Identification of Ablation Sites in Persistent Atrial Fibrillation Based on Spatiotemporal Dispersion of Electrograms Using Machine Learning
}

\author{
Amina Ghrissi ${ }^{1}$, Fabien Squara ${ }^{1,2}$, Johan Montagnat ${ }^{1}$ and Vicente Zarzoso ${ }^{1}$ \\ ${ }^{1}$ Université Côte d'Azur, CNRS, I3S Laboratory, Sophia Antipolis, France \\ ${ }^{2}$ Université Côte d'Azur, CHU Pasteur, Cardiology Department, Nice, France
}

\begin{abstract}
A recent patient-tailored ablation protocol to treat atrial fibrillation consists in identifying ablation sites based on their spatiotemporal dispersion (STD). STD represents a delay of the cardiac activation observed in intracardiac electrograms (EGMs) across contiguous leads. This work aims at automatically identifying ablation sites by classifying EGM data acquired by the PentaRay catheter into ablated vs. non-ablated groups using machine learning. More than 35000 multichannel recordings are acquired from 15 persistent AF patients. An annotation model is designed to label the dataset. The classifiers include: (1) multivariate logistic regression; (2) LeNet-STD, a shallow convolutional neural network. A binary label identifying whether the mapped site contains STD pattern according to the interventional cardiologist is combined to raw EGMs as classifiers input. The LeNet-STD combined with data augmentation yields the best performance with an F1score of $76 \%$.
\end{abstract}

\section{Introduction}

Atrial fibrillation (AF) represents the most widespread sustained arrhythmia experienced in clinical practice rising in prevalence with advancing age. $\mathrm{AF}$ is characterized by an irregular activation in the atria that start quivering causing the ventricular rate to become more rapid and disorganized [1]. Among the existing treatments for persistent $\mathrm{AF}$, ablation interventions overperform drug therapies in terms of efficiency.

Ablation is an invasive procedure that consists in burning the cardiac tissues displaying irregularities with RF energy delivered through catheters. Classical ablation protocol is called stepwise and consists in: 1) burning the triggers around the pulmonary veins thought to be responsible for initiating AF; 2) ablating cardiac areas exhibiting complex fractionated electrograms [2]. However, a growing number of reports show the limitations of the stepwise approach [3].
A novel wholly patient-tailored ablation protocol, giving $95 \%$ of procedural success rate, consists in identifying ablation sites based on a pattern called spatiotemporal dispersion (STD) [4]. Multipolar mapping catheters are used to record electrograms (EGMs) in the atria thus targeting areas of STD as potential AF drivers. The high-density mapping PentaRay catheter (Biosense Webster Inc, Irvine, CA, USA) is used for STD localization. It has a five-branch star design with two bipoles on each spline.

Before ablation with RF energy, interventional cardiologists first position sequentially the PentaRay in different atrial sites. Ten bipoles are then simultaneously recorded per location by maintaining the catheter stable for at least $2.5 \mathrm{~s}$. Finally, atrial sites displaying an irregular cardiac activity are annotated as dispersion points and tagged for ablation. According to guidelines in [4], dispersion areas refer to clusters of electrograms, either fractionated or not, displaying interelectrode time and space dispersion at a minimum of three contiguous leads, as shown in Fig. 1. Hence, STD-based ablation is a fully patient-tailored therapy. According to preliminary guidelines for STD identification from visual inspection, the 10-channel EGM recording acquired by the PentaRay would display a cardiac activation delay of $70 \%$ of $\mathrm{AF}$ cycle length (AFCL) on a minimum of three neighboring bipoles (channels) [4]. A recent study proposed to automatically detect STD EGMs using machine learning (ML) [5] and benchmarked a set of adapted data augmentation (DA) approaches to handle the high class imbalance ratio between STD and non-STD classes [6].

The present contribution proposes to design a decisionaid solution that helps interventional cardiologists identify potential ablation sites automatically using ML tools. We classify ablated vs. non-ablated mapped sites using: (1) multivariate logistic regression (MLR); (2) LeNetSTD, a shallow convolutional neural network (CNN). A binary label identifying whether the mapped site contains STD pattern according to the interventional cardiologist is combined to raw EGMs as classifiers input. This additional input can be assimilated to a prior probability in 
STD-guided ablation. Data augmentation is used to handle the dataset imbalance. ML techniques allow to automatically identify ablated sites and guide cardiologists in patient-tailored catheter ablation procedures for treating persistent AF.

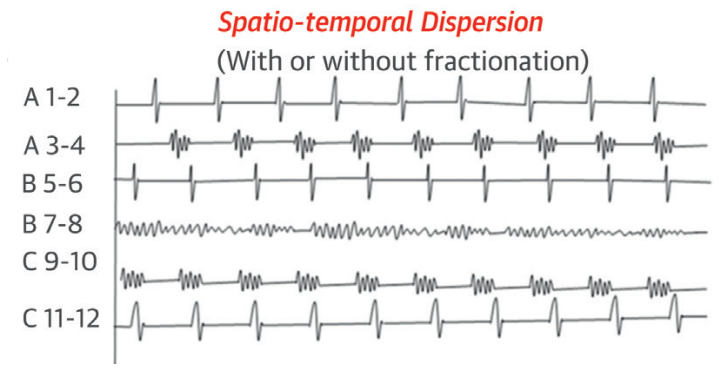

Figure 1. Dispersion areas are defined and delineated via a mapping approach [4]. Channels A 1-2, A 3-4, B 5-6, B 7-8, C 9-10 and C 11-12 display STD.

\section{Methods}

\subsection{Study dataset}

Data are provided by the Cardiology Department of Nice University Hospital Center (CHU) and are exported from the BioSense CARTO system. This intervention data come from 15 patients ablated for persistent AF based on STD pattern with the use of PentaRay catheter, shown in Fig. 2. They include electrocardiogram, EGM and anno-

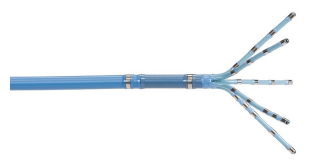

Figure 2. PentaRay multi-spline catheter.

tated dispersion points from both right and left atrial cartographies. The study dataset includes a cohort of 35563 10-channel EGM signals of length $2.5 \mathrm{~s}$ composed of 1804 samples labeled STD and 33759 non-STD samples. Data annotation is performed by interventional cardiologists such that EGMs presenting spatiotemporal dispersion are labeled as 'STD'. We automatically merge the remaining labels into the 'non-STD' class.

\subsection{Annotation model}

The intervention is a two-fold process: 1) mapping the atria and tagging STD sites; 2) ablating AF drivers (atrial locations) that have not necessarily been mapped in advance. An annotated dataset is then needed to train supervised ML classifiers that automatically detect the ablated mapped points among all the mapped ones (including STD and non-STD). The labels are binary and should inform if the mapped point (data sample) has been ablated or not. This information is not provided by the CARTO system because: 1) the mapping and ablation phases are decoupled; 2) the ablation sites are not restricted to the mapped points tagged as STD. Indeed, there might be STD positions that are not ablated probably because they are isolated and do not form a cluster or because of anatomical reasons. Besides, there might be non-STD positions that are ablated because they are close enough to STD clusters or for anatomical reasons. For this purpose, we design a labeling model based on the proximity between both mapped $\mathcal{P}$ and ablated $\mathcal{V}$. Due to the limited precision of the ablation catheter and the manual ablation process, the catheter might be slightly misplaced around the desired position $(D)$. In practice, the catheter may slightly move around the desired position $D$ during an ablation shot, also called a session. $D$ might correspond or not to a mapped point $P$.

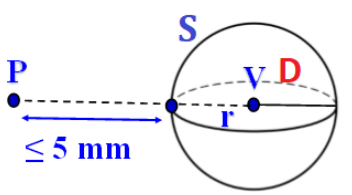

Figure 3. Sphere model of the ablation site.

Based on these observations, we propose to consider each area ablated around $D$ as a sphere $(\mathcal{S})$ of center $V$ and radius $r$. The Cartesian coordinates of $V$ correspond to the average Cartesian coordinates of the points visited by the ablation catheter during the ablation session and $r$ is the square root of the standard deviations of the corresponding coordinates. According to the partner interventional cardiologist, the distance between a mapped point $P$ and the closest ablation zone $\mathcal{S}$ should be smaller or equal to $5 \mathrm{~mm}$ for $P$ to be considered as ablated. This can be mathematically translated into $\|\overrightarrow{P V}\|_{2}-r \leq 5$. Hence, for each ablation session, the mapped points that lie at most $5 \mathrm{~mm}$ away from $\mathcal{S}$ are attributed the 'ablated' label $\left(P_{a b}\right)$, resulting in the set:

$$
\mathcal{P}_{a b}=\left\{P_{i} \in \mathcal{P}: \exists V_{j} \in \mathcal{V}, d\left(P_{i}, V_{j}\right) \leq 5+r_{j}\right\}
$$

Mapped points not fulfilling Eq. (1) form the non-ablated class $\left(P_{\text {non-ab }}\right)$. Based on this model, we obtain 15803 ablated and 19760 non-ablated points.

\subsection{Classification algorithms}

Logistic regression model estimates the probability of a given class by using the logistic function. MLR is a baseline classifier in biomedical data analysis [7].

CNNs are commonly used in biomedical data classification [8]. A CNN is composed of convolutional (conv) 
and pooling layers followed by fully connected (FC) ones. LeNet-STD is a CNN inspired by LeNet-5 architecture [9] which represents a good and computationally-affordable model. Let $Z$ and kernel respectively refer to the number of filters and the receptive field of the filters. LeNet-STD is composed of the following layers: 1) 2-dimensional (2D) conv ( $Z=32$, kernel $=4 \times 300) ; 2$ ) average pooling; 3 ) $2 \mathrm{D}$ conv $(Z=16$, kernel $=3 \times 3)$; 4) average pooling; 5) FC $(Z=128) ; 6) \mathrm{FC}(Z=64)$; 7) final FC decision $(Z=2)$.

During the mapping phase, the PentaRay catheter is maintained stable for $2.5 \mathrm{~s}$ at each location. A location refers to anatomical point inside the heart. Multichannel EGMs are sampled at $1 \mathrm{kHz}$, recorded in the hospital's database then displayed through the CARTO system monitor, to be analyzed by the cardiologist. Hence, EGM samples can be stored in a matrix of dimension $10 \times 2500$ and classified as images.

\subsection{Data Augmentation}

DA applies transformations to original samples of the minority class in order to synthesize new samples. A recent study proved that random oversampling (ROS) is the best technique for the classification of multichannel EGMs into STD vs. non-STD [6]. ROS forms a balanced superdataset by randomly replicating STD samples until they reach the number of the non-STD ones.

\section{Experiments and Results}

Circularity transformation is applied to each data sample in order to mimic the circularity of PentaRay branches. This transformation consists in replicating the first two rows of the $10 \times 2500$ matrix at its end to form a matrix of dimensions $12 \times 2500$ [6].

In order to identify potential ablation sites in STD-based ablation of persistent AF, we train baseline classifiers including MLR and LeNet-STD. The study dataset is annotated according to the model in Sec. 2.2 and is composed of 15803 ablated $\left(P_{\mathrm{ab}}\right)$ and 19760 non-ablated $\left(P_{\text {non-ab }}\right)$ samples.

We conduct three types of experiments:

1) We train LeNet-STD and MLR with mini-batch gradient descent and early stopping criteria. The receptive field of the first convolutional layer of LeNet-STD is chosen large enough to cover the information between all three contiguous leads along one AFCL and a half thus fully capturing potential STD patterns.

2) The class imbalance ratio between labeled and nonlabeled classes is equal to $\frac{\# P_{\mathrm{ab}}}{\# P_{\text {non-ab }}}=80 \%$, where $\# A$ refers to the cardinality of the set $A$. As a result, classes are slightly imbalanced and the classifiers might face problems learning characteristics from the minority class (ablated) due to the insufficient number of $P_{\mathrm{ab}}$ samples.We propose to use a DA method called ROS that proved efficient in [6]. The resulting models are denoted MLR-ROS and LeNet-ROS.

3) Based on the fact that both ablated and STD atrial areas should be spatially correlated, we concatenate the information about whether a data point is labeled STD or not with the previous classifiers' output. This additional input can be assimilated to a prior probability in STDguided ablation. We build new architectures for MLR and LeNet-STD, denoted MLR-STDlab and LeNet-STDlab, by adding a sub-neural network (sub-NN) composed of a dense FC layer. The sub-NN gets the STD label as input and outputs a score function that will be concatenated with the output of the final decision layer of MLR and LeNet.

Five-fold cross-validation (CV) is used to asses the generalization power of the trained classifiers. The classification results shown in Figures 4(a) and 4(b) represent the average performance on the test dataset across the 5 folds of MLR and LeNet respectively. The metrics computed are: 1) accuracy (Acc); 2) area under the ROC curve (AUC); 3) sensitivity, also called true positive rate (TPR) or true rate of ablated samples; 4) specificity also called true negative rate (TNR) or true rate of non-ablated samples; 5) precision, also called positive predictive value (PPV); 6) negative predictive value (NPV); 7) F1-score. The results show that the overall performance of LeNet-STD improves than that of MLR with values of Acc, AUC and F1-score respectively equal to $75 \%, 82 \%, 73 \%$ vs. $72 \%, 78 \%, 67 \%$. ROS allowed us to increase the TPR from $76 \%$ to $84 \%$ while maintaining TNR at $70 \%$ which further increases the F1-score to $76 \%$ with LeNet-STD architecture. Concatenating with STD labels did not help improve neither decrease the classification performance. Also, the standard deviation of the classification metrics through $\mathrm{CV}$ does not exceed $10^{-2}$. We conclude then that LeNet-STD-ROS is the best model.

\section{Conclusions and perspectives}

The automatic identification of potential ablations sites in STD-based ablation of persistent AF can help guide interventional cardiologists in patient-tailored procedures. For this task, we design a model to annotate the multichannel EGM dataset into ablated vs. non-ablated classes based on Euclidean distance between mapped and ablated points. ML tools are used to classify the labeled samples into ablated vs. non-ablated categories. MLR and LeNetSTD are benchmarked whether combined with DA or not. Moreover, we concatenate the supervised classifiers with sub-NN that takes as input the label of whether the considered data sample is tagged STD or non-STD. Five-fold $\mathrm{CV}$ is also used to asses the robustness of trained models. Classification results on the test set show that LeNet-STD combined with ROS give the best performance with an 

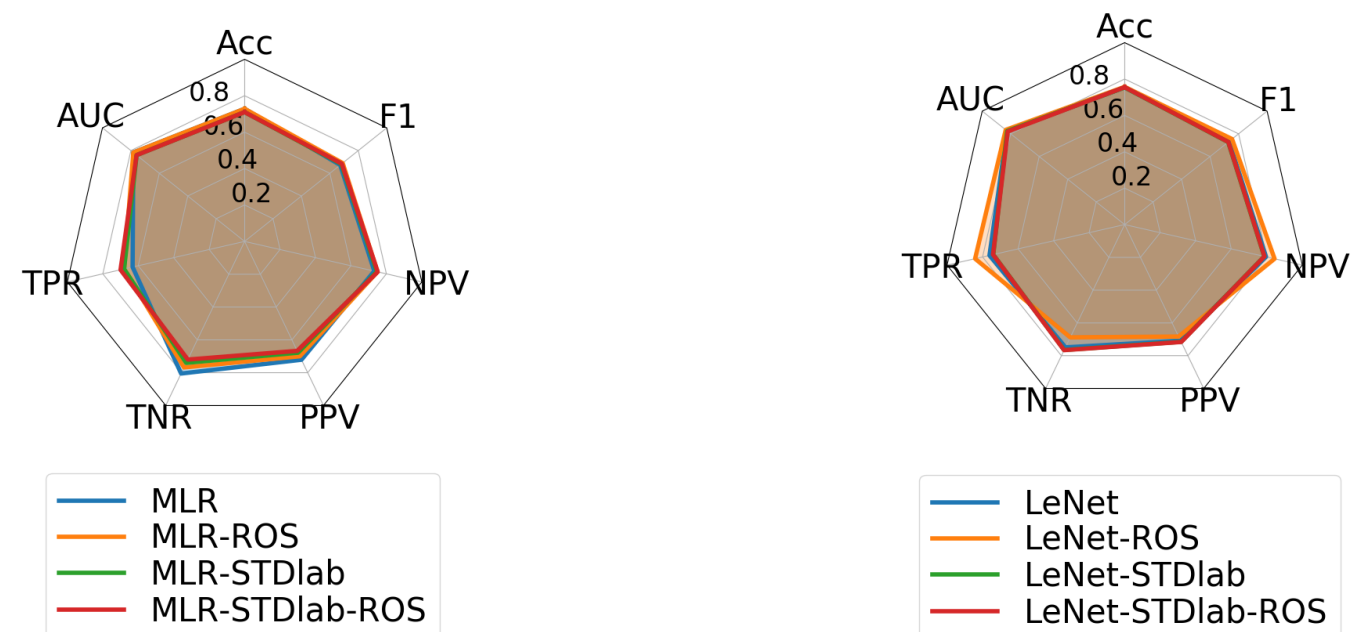

Figure 4. Classification performance on the test dataset. Values represent averages through 5-fold CV. Left: MLR classifier and variants. Right: LeNet-STD classifier and variants.

F1-score of 76\%. Aggregating STD label did not help improve the model's performance. This can be interpreted by the fact that ablation takes into consideration more anatomical factors than the STD pattern. Moreover, the sub-NN used to analyze STD labels is shallow and might be not efficient. Future work should aim at designing deeper architectures that might take further advantage of STD information to improve the automatic detection of ablation sites.

\section{Acknowledgments}

This work is funded by the French government PIA program, IDEX UCA ${ }^{\text {JEDI }}$ project (ANR-15-IDEX-0001).

\section{References}

[1] C. T. January, et al. "2014 AHA/ACC/HRS guideline for the management of patients with atrial fibrillation," Journal of the American College of Cardiology, 64(21), 2014.

[2] K. Nademanee, et al. "A new approach for catheter ablation of atrial fibrillation: mapping of the electrophysiologic substrate," Journal of the American College of Cardiology. Journal of the American College of Cardiology, 43(11), pp. 2044-2053, 2004.

[3] A. Verma, et al. "Approaches to catheter ablation for persistent atrial fibrillation," New England Journal of Medicine, 372(19), pp. 1812-1822, 2015.

[4] J. Seitz, et al. "AF ablation guided by spatiotemporal electrogram dispersion without pulmonary vein isolation: a wholly patient-tailored approach," Journal of the American College of Cardiology, 69(3), pp. 303-321, 2017.

[5] I. Goodfellow, et al. Deep Learning, MIT Press, 2016. www.deeplearningbook.org.
[6] A. Ghrissi, et al. "Data Augmentation for Automatic Identification of Spatiotemporal Dispersion Electrograms in Atrial Fibrillation Ablation Using Machine Learning," in $42^{\text {nd }}$ Annual International Conference of the IEEE Engineering in Medicine and Biology Society, EMBC 2020, Montreal, 2020.

[7] S. Dreiseitl, O. M. Lucila. "Logistic regression and artificial neural network classification models," Journal of Biomedical Informatics, 35(5-6), pp. 352-359, 2002.

[8] B. Pyakillya, et al. "Deep learning for ECG classification," Journal of Physics, 913(1), IOP Publishing, 2017.

[9] Y. LeCun, et al. "Gradient-based learning applied to document recognition," Proceedings of the IEEE, 86(11), pp. 2278-2324, 1998.

Address for correspondence:

Amina Ghrissi

Université Côte d'Azur, CNRS, I3S Laboratory

Les Algorithmes, Euclide B, 06103, Sophia Antipolis, France

amina.ghrissi@univ-cotedazur.fr 